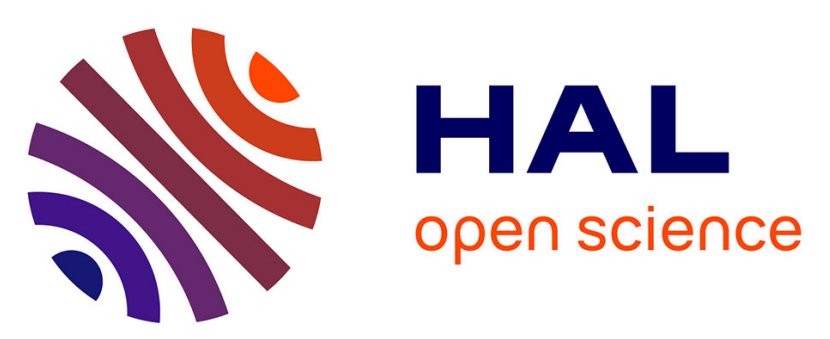

\title{
Terminal Base Pairs of Oligodeoxynucleotides: Imino Proton Exchange and Fraying
}

\author{
Sylvie Nonin, Jean-Louis Leroy, Maurice Guéron
}

\section{To cite this version:}

Sylvie Nonin, Jean-Louis Leroy, Maurice Guéron. Terminal Base Pairs of Oligodeoxynucleotides: Imino Proton Exchange and Fraying. Biochemistry, 1995, 34 (33), pp.10652-10659. 10.1021/bi00033a041 . hal-02344774

\section{HAL Id: hal-02344774 \\ https://hal.science/hal-02344774}

Submitted on 11 Aug 2020

HAL is a multi-disciplinary open access archive for the deposit and dissemination of scientific research documents, whether they are published or not. The documents may come from teaching and research institutions in France or abroad, or from public or private research centers.
L'archive ouverte pluridisciplinaire HAL, est destinée au dépôt et à la diffusion de documents scientifiques de niveau recherche, publiés ou non, émanant des établissements d'enseignement et de recherche français ou étrangers, des laboratoires publics ou privés. 


\title{
Terminal Base Pairs of Oligodeoxynucleotides: Imino Proton Exchange and Fraying $^{\dagger}$
}

\author{
Sylvie Nonin, ${ }^{\neq, 8}$ Jean-Louis Leroy, ${ }^{8}$ and Maurice Guéron $* .8$ \\ Groupe de Biophysique de l'Ecole Polytechnique et de l'URA DI254 du CNRS, 91128 Palaiseau, France, and \\ CEA/SBGM, Centre d'Etudes de Saclay 91191 Gif-sur-Yvette, France
}

\begin{abstract}
We have estimated the dissociation constant of the terminal base pairs of the B-DNA duplexes formed by $5^{\prime}$-d(CGCGATCGCG) and $5^{\prime}$-d(TAGCGCTA) by two methods, one based on the change in imino proton chemical shift with temperature and the other on the apparent $\mathrm{p} K$ shift of the imino proton, as monitored by the change in chemical shift of aromatic protons. These methods do not rely on imino proton exchange, whose rate was also measured. (1) The effect of ammonia on the imino proton exchange rate of the terminal pair of the $5^{\prime}$-d(CGCGATCGCG) duplex is 67 times less than on the isolated nucleoside. This provides an upper limit on the exchange rate from the closed pair. In fact, the effect is just as predicted from the dissociation constant, assuming that there is no exchange at all from the closed pair and that, as has been argued previously, external catalysts act on the open state as they do on the isolated nucleoside. The inhibition of catalyzed proton exchange in the closed pair, despite exposure of one face of the pair to solvent, is a new feature of the exchange process. It will allow determination of the dissociation constant of terminal pairs from the exchange rate. (2) Intrinsic catalysis of proton exchange is less efficient for the terminal pair than for an internal one. A possible explanation is that proton transfer across the water bridge responsible for intrinsic catalysis is slower, as expected if the open-state separation of the bases is larger in a terminal pair. This observation may lead to a direct method for the study of fraying. (3) At $0^{\circ} \mathrm{C}$, the dissociation constant of the second pair of the $5^{\prime}-\mathrm{d}$ (CGCGATCGCG) duplex is close to the square of the constant for the terminal pair, as predicted from a simple model of fraying. The enthalpy and entropy of opening of the terminal pairs may be compared with those of nearest neighbor interactions derived from calorimetry [Breslauer, K. J., et al. (1986) Proc. Natl. Acad. Sci. U.S.A. 83, $3746-3750]$.
\end{abstract}

Imino proton exchange from an internal Watson-Crick base pair of a nucleic acid duplex is fairly well characterized. It requires opening of the pair. In the open state, external exchange catalysts act in similar fashion as on the isolated nucleoside. From the effect of the catalyst, one derives the base pair dissociation constant and, by extrapolation of the exchange time to infinite catalyst concentration, the lifetime of the closed pair (Guéron \& Leroy, 1995). With this understanding, one may explore the base pair kinetics of short duplexes and show, for instance, that base pairs open one at a time. The kinetic data also provide information on the duplex structure, e.g., in the case of adenine tracts (Leroy et al., 1988a) and on the open state of the base pair. The latter includes an intrinsic exchange catalyst, the acceptor imino nitrogen of A or C (Guéron et al., 1987). Its operation requires the presence of an indirect $\mathrm{H}$-bond, via a water bridge, between the two bases of the open pair, a constraint on the opening process and on the geometry of the open state.

However, our knowledge of proton exchange is still incomplete, and this limits its applications. Consider for instance the wobble pair G.T. The two imino protons are

\footnotetext{
'This study was supported by a grant to M.G. from the Association pour la Recherche contre le Cancer. Support to S.N. was provided by Ecole Polytechnique and by Commissariat à l'Energie atomique.

${ }^{\ddagger} \mathrm{CEA} / \mathrm{SBGM}$.

CNRS.
}

accessible sideways, from within the base plane. Does this enable exchange from the closed pair? Lacking an answer to this question, we cannot interpret the proton exchange data in terms of internal motions (Guéron \& Leroy, 1992). Another example is the kink, with two base pairs unstacked so that one face of each pair may be accessible to the solution. If the imino proton exchanged from the closed pair in such a situation, one could use proton exchange to study kinking. A third problem, for which a solution may now be in view (S. Nonin, J. L. Leroy and M. Guéron, manuscript in preparation), is the extremely efficient mechanism of acid catalysis of imino proton exchange from $\mathrm{G} \cdot \mathrm{C}$ pairs (Guéron \& Leroy, 1992). As a last example, consider the exchange of amino protons, which is not fully understood even in the case of isolated nucleosides.

In the present work, we study exchange from the terminal base pair of a duplex. Like the pairs at a kink, the terminal pair has one face exposed to the solution. We investigate whether the imino proton exchanges from the closed pair. We measure the exchange rate at moderate catalyst concentrations and compare it to the rate predicted for exchange from the open state only. The difference between rates, if any, is assigned to exchange from the closed pair.

This approach requires a measurement of the dissociation constant of the terminal pair by methods which are independent of the exchange process. Two such methods are proposed. Melting of the base pair is traced by the variation 
of the chemical shift of the imino proton $v s$ temperature; the dissociation constant is derived from the apparent $\mathrm{p} K$ shift of the imino proton, traced by the titration of the aromatic proton spectrum.

We use for these studies the self-complementary B-DNA duplexes [5'-d(CGCGATCGCG) $]_{2}$ and [5'-d(TAGCGCTA) $]_{2}$. Of the two duplexes, the first one has the terminal C.G pair with the smallest dissociation constant, offering therefore the most severe test of exchange from the closed pair. The second duplex provides a case of a terminal $\mathrm{T} \cdot \mathrm{A}$ pair.

The present work provides new methods for the study of the dissociation of terminal pairs. The values thus obtained should be considered in relation to the nearest-neighbor interactions determined by calorimetry (Breslauer et al., 1986).

\section{MATERIALS AND METHODS}

Deoxyoligonucleotides. The oligomers were synthetized by the $\beta$-cyanoethyl phosphoramidite method on a $10 \mu \mathrm{M}$ scale. Cleavage and deprotection were carried out overnight in $25 \%$ aqueous ammonia at $60^{\circ} \mathrm{C}$. Samples were purified by high-pressure chromatography as previously described (Kochoyan et al., 1990), filtered (Millipore 0.22- $\mu \mathrm{m}$ filter), and dialyzed successively against $10 \mathrm{mM} \mathrm{NaCl}$ solutions and against pure water, using plastic containers. The dialysate was then lyophilized. The NMR line widths were not reduced by $2 \mathrm{mM}$ EDTA, indicating that contamination by paramagnetic ions was negligible.

NMR Sample Preparation. Samples (100 OD units, $A_{260}$ $\mathrm{nm}$ ) were dissolved in $400 \mu \mathrm{L}$ of a solution of $\mathrm{NaCl}$ in $97.5 \%$ $\mathrm{H}_{2} \mathrm{O}-2.5 \% \mathrm{D}_{2} \mathrm{O}$ and $2 \mathrm{mM}$ EDTA. Tetramethylammonium chloride was used as the chemical shift reference. The $\mathrm{pH}$ was measured with a glass microelectrode and adjusted with $0.2-1-\mu \mathrm{L}$ volumes of filtered stock solutions $(0.006-6 \mathrm{M})$ of $\mathrm{HCl}$ or $\mathrm{NaOH}$. The sample volume was reduced when necessary by surface evaporation in a current of nitrogen.

NMR Experiments. $1 \mathrm{D}^{\mathrm{l}}$ spectra were obtained on an 8.7T, 360-MHz home-built NMR spectrometer. The sample volume was usually $400 \mu \mathrm{L}$. For $50-100-\mu \mathrm{L}$ volumes, a glass plug was held above the solution. We used the poweradapted jump-and-return (PJR) excitation sequence for solvent signal suppression (Guéron et al., 1991) and composite DANTE pulses at the water frequency for selective excitation (Morris \& Freeman, 1963). Residual transverse magnetization of water was destroyed if necessary by a 5-10-ms z-gradient pulse. For small samples, the gradient was not required and the minimum delay after selective inversion of water was $1 \mathrm{~ms}$. The free precession was processed with exponential multiplication, and Hamming apodization as required. The residual water signal was reduced by repetitive simulation and subtraction of Lorentzians from the experimental spectrum. The effect of exchange catalysts was derived from the variation of either the longitudinal relaxation rate, the line width (in cases where $\left.T_{1}<1 \mathrm{~ms}\right)$, or the rate of magnetization transfer $\left(T_{1}>50\right.$ $\mathrm{ms}$ ) (Leroy et al., 1988b).

The study of the terminal imino proton may require special methods, due to large line width or spectral overlap. In such

\footnotetext{
'Abbreviations: 1D, one-dimensional; 2D, two-dimensional; FID, free induction decay; NOE, nuclear Overhauser effect; NOESY, nuclear Overhauser effect spectroscopy; TPPI, time proportional phase increment.
}

cases, the excitation method included selective inversion of the imino proton spectrum, a delay $(15 \mathrm{~ms})$ shorter than the relaxation time of the internal imino protons but longer than that of the terminal one, and a PJR observation sequence. The acquisition time was also short (e.g., $20 \mathrm{~ms}$ ), and the sequence was repeated with no waiting time. This procedure results in efficient observation of the terminal imino proton, while the internal ones are highly saturated, as can be seen in Figure 3A.

2D NOESY spectra were recorded at 10 and $-7{ }^{\circ} \mathrm{C}$ in $\mathrm{D}_{2} \mathrm{O}$ on a Bruker AMX $600-\mathrm{MHz}$ spectrometer in the TPPI phase cycling mode, using a $t_{2}$ acquisition time of $250 \mathrm{~ms}$ (2048 data points, 136 acquisitions) and a $t_{1}$ range of 31 $\mathrm{ms}$. The mixing time was $400 \mathrm{~ms}$. The residual DHO signal was suppressed by presaturation. In both dimensions, the data were multiplied by a sine bell shifted by $\pi / 8$ before Fourier transformation.

Spectral Assignments. The internal imino protons were assigned by $1 \mathrm{D}$ and 2D imino-imino proton NOE in $\mathrm{H}_{2} \mathrm{O}$. The terminal imino proton was assigned as the remaining one, or by its large line width, its short $T_{1}$, and/or its large variation of chemical shift with temperature (see Results).

The aromatic protons of the d(TAGCGCTA) duplex were assigned by $\mathrm{D}_{2} \mathrm{O}$ NOESY spectra (400-ms mixing time). The sequential connectivities were analyzed assuming a B-DNA structure, and they support that assumption. They could be traced along the entire strand. The assignments were carried out at high $\mathrm{pH}$, so as to split the $\mathrm{H} 8$ peaks of the two adenines (downfield from those of $\mathrm{G}$ ) and the $\mathrm{H} 6$ peaks of the two thymines. A cross-peak at $(7.80,2.67) \mathrm{ppm}$ was assigned to $\mathrm{GH} 8 / \mathrm{AH} 2$ " and used as the starting point in the assignment procedure.

The spectrum of the $5^{\prime}$-d(CGCGATCGCG) duplex has been assigned previously (Leroy et al., 1988; Patel et al., 1991).

pH Measurements. For the studies at room temperature and at $0{ }^{\circ} \mathrm{C}, \mathrm{pH}$ and $\mathrm{pD}$ were determined by measurement at the temperature of the experiment. The $\mathrm{pD}$ value was set equal to the indicated value +0.4 .

For studies of the imino proton shift at other temperatures (Figure 3), the $\mathrm{pH}$ was measured at room temperature. It varies slightly with temperature, but the variation is of no consequence for this experiment. For the $-7{ }^{\circ} \mathrm{C}$ experiments also, the $\mathrm{pH}$ is measured at $20^{\circ} \mathrm{C}$. The change of $\mathrm{pH}$ between the two temperatures depends on the buffering capacity of the nucleic acid, and it may therefore differ between the duplex and nucleoside solutions. As a result, these data cannot be used for a precise comparison of the exchange rates of the terminal pair and of the nucleoside (Figure 1).

\section{THEORY}

Proton Exchange. The theory of catalyzed proton exchange has been described (Eigen, 1964; Guéron et al., 1990). In the case of internal base pairs of a duplex (i.e., pairs which are not terminal), exchange does not occur unless the pair is open. External catalysts act similarly on the open state and on the isolated nucleoside. In the open state, there is also an intrinsic catalyst: the nitrogen acceptor of the imino $\mathrm{H}$-bond. The exchange process may be limited by basepair opening or by exchange catalysis. When the base pair dissociation constant $K_{\text {diss }}$ is much less than 1, as in the conditions of our exchange measurements, the kinetics is first 
order, and one has

$$
\tau_{\mathrm{ex}}=1 / k_{\mathrm{ex}} \approx \tau_{0}+\left(1+1 / K_{\mathrm{diss}}\right) \tau_{\mathrm{ex}, \mathrm{open}}
$$

where $\tau_{\text {ex }}$ and $1 / k_{\text {ex }}$ are the exchange time and rate, $\tau_{0}$ is the base pair lifetime, and $\tau_{\text {ex,open }}$ is the exchange time in the open state, the inverse of the exchange rate $k_{\text {ex.open }}$.

In the case of an external catalyst such as $\mathrm{OH}^{-}$or $\mathrm{NH}_{3}$, one sets

$$
k_{\text {ex,open }}^{\text {ext }}=\alpha k_{\mathrm{i}}
$$

where $k_{\mathrm{i}}$ is the exchange rate of the isolated nucleoside in the same conditions, which is proportional to the catalyst concentration. It is measured separately. The coefficient $\alpha$ encompasses the differences between external catalysis in the open state and in the case of the isolated nucleoside. As discussed elsewhere, the $\alpha$ values for different catalysts are not too different, nor are they too far from unity (Guéron $e t$ al., 1990).

A second contribution to exchange from the open state is that due to the intrinsic catalyst, $k^{\text {int }}$ ex,open (Guéron et al., 1987). It adds to the contribution of the external catalyst. The net exchange rate is then

$$
\tau_{\mathrm{ex}} \equiv 1 / k_{\mathrm{ex}} \approx \tau_{0}+\left(1+1 / K_{\mathrm{diss}}\right) /\left(\alpha k_{\mathrm{i}}+k_{\text {ex }, \mathrm{open}}^{\mathrm{int}}\right)
$$

A plot of the exchange time, corrected for the effect of intrinsic catalysis, $v s$ the inverse of the catalyst concentration is a straight line which extrapolates to $\tau_{0}$ for infinite catalyst concentration. For the isolated nucleoside, the extrapolation is to zero. The ratio of the slopes for nucleoside and base pair is equal to $\alpha /\left(1+1 / K_{\text {diss }}\right)$. The expression $\alpha K_{\text {diss }}$ is called the apparent dissociation constant.

In the case of a terminal base pair, one face of the pair is exposed to the solution, and the possibility of proton exchange from the closed pair must be considered. If the dissociation constant is much smaller than 1 , the exchange is still first order, and its rate $k_{\text {ex }}$ is

$$
k_{\mathrm{ex}}^{\prime} \approx k_{\mathrm{ex}}^{\mathrm{cl}}+k_{\mathrm{ex}}
$$

where $k_{\mathrm{ex}}{ }^{\mathrm{cl}}$ is the rate of exchange from the closed state, and $k_{\text {ex }}$ is the contribution from exchange in the open state (eq 3).

Effect of Base Pair Opening on the Chemical Shift of the Imino Proton. Proton exchange between the imino proton position and the solvent broadens but does not shift the imino proton resonance, because the proton leaving the imino site never comes back to it. In contrast, the alternation between closed and open states produces a shift, but usually no appreciable broadening because the open-state lifetime is so short. The shift $\delta$ is the average of the shifts $\delta_{\text {closed }}$ and $\delta_{\text {open }}$ in the open and closed states, weighed by their relative populations:

$$
\begin{gathered}
\left(\delta_{\text {closed }}-\delta\right) /\left(\delta-\delta_{\text {open }}\right)=K_{\text {diss }} \\
\delta=\delta_{\text {closed }} /\left(1+K_{\text {diss }}\right)+K_{\text {diss }} \delta_{\text {open }} /\left(1+K_{\text {diss }}\right)
\end{gathered}
$$

In the case of the terminal base pair, the large dissociation constant leads to a measurable shift, from which the dissociation constant can be derived, independently of exchange kinetics, and without interference from the process of exchange with water.
Titration of a Paired Base: The Apparent $p K$ Shift. The $\mathrm{p} K$ of the imino proton of a nucleoside, $\mathrm{p} K_{\mathrm{i}}$, is easily determined by titration of the chemical shift of an aromatic proton, for instance, the $\mathrm{H} 8$ proton of deoxyguanosine. In a base pair, the midtitration point is changed. This is a Le Châtelier displacement of equilibrium, due simply to the fact that $\mathrm{G}$ (or $\mathrm{T}$ ) in the closed pair is necessarily protonated, so that one is dealing with a three-species system: protonated $\mathrm{G}$ in the closed pair (designated $\mathrm{c}$ ), protonated $\mathrm{G}$ in the open pair (o), and de-protonated $\mathrm{G}$ in the open pair $\left(\mathrm{o}^{-}\right)$. We use the same symbols for the relative proportions of these species. The opening of the pair takes place without deprotonation, so that the constant $K_{\mathrm{d}}$ of the corresponding equilibrium (c $\leftrightarrow$ o) is pH-independent. In contrast, the two open-pair species, $\mathrm{o}$ and $\mathrm{o}^{-}$, are in the ratio $10^{\mathrm{p} K_{\mathrm{i}}-\mathrm{pH}}$. The midtitration $\mathrm{pH}$ is equal to an apparent $\mathrm{p} K$, denoted $\mathrm{p} K$ (app) and given by

$$
(\mathrm{o}+\mathrm{c}) / \mathrm{o}^{-} \equiv\left(\mathrm{o} / \mathrm{o}^{-}\right)(1+\mathrm{c} / \mathrm{o}) \equiv 10^{\mathrm{p}^{K_{\mathrm{i}}-\mathrm{pH}}}\left(1+1 / K_{\mathrm{d}}\right)=1
$$

Hence

$$
10^{\mathrm{p} K(\mathrm{app})-\mathrm{p} K_{\mathrm{i}}}=\left(1+1 / K_{\mathrm{d}}\right)
$$

The measurement of the $\mathrm{p} K$ shift thus provides a second method for determining the dissociation constant independently of the exchange rate.

The dissociation constant is a function of $\mathrm{pH}$

$$
K_{\text {diss }} \equiv\left(\mathrm{o}^{-}+\mathrm{o}^{-}\right) / \mathrm{c} \equiv(\mathrm{o} / \mathrm{c})\left(1+\mathrm{o}^{-} / \mathrm{o}\right)=K_{\mathrm{d}}\left(1+10^{\mathrm{pH}-\mathrm{p} K_{\mathrm{j}}}\right)
$$

The relative proportions of the three species, $\mathrm{o}^{-}$, and $\mathrm{c}$, vary with $\mathrm{pH}$, but it is noteworthy that for $\mathrm{K}_{\mathrm{d}} \ll 1$ the fraction of o is always small as compared to 1 : when $\mathrm{pH}$ is less than $\mathrm{p} K_{\mathrm{spp}}$, the pair is mostly closed; and when $\mathrm{pH}$ increases to $\mathrm{p} K_{\mathrm{upp}}$ and beyond, the fraction of $\mathrm{o}^{-}$is larger than that of o, because $\mathrm{p} K_{\text {app }}$ is larger than $\mathrm{p} K_{\mathrm{i}}$.

Fraying. At low temperature and neutral pH, the dissociation constant of the terminal pair is usually rather small, and that of the second pair is smaller still. But at higher temperature and $\mathrm{pH}$, the terminal pair is open a large part of the time. When this happens, the second pair is in a quasiterminal pair situation, and it also opens up. We have moved from a two-state to a four-state problem, and the experimental constraints are far too few to solve it without simplifications. In the d(CGCGATCGCG) duplex, each of the terminal and second pairs is a $\mathrm{G}^{*} \mathrm{C}$ pair resting on a $\mathrm{C} \cdot \mathrm{G}$ pair. We therefore assume that the dissociation constant of the second pair, when the first is open, is the same as that of the first pair. We also assume that the chemical shift of the imino proton of the open terminal pair is the same, whether the second pair is open or closed.

We designate by $\mathrm{c} 1 \mathrm{c} 2$ the situation where the two pairs are closed, by olc2 (respectively $\mathrm{ol}^{-} \mathrm{c2}$ ) the situation where the first is open and protonated (respectively unprotonated) and the second one closed, and so on. When the first pair is closed, we expect the second one to have the very small dissociation constant typical of an internal pair. We therefore consider only three situations, c1c2, o1c2, and olo2, and their unprotonated relatives. We use the same symbols to represent their relative concentrations. In analogy with the definition of $K_{\mathrm{d}}$ above, we define $K_{\mathrm{d}}(1 ; \mathrm{c} 2)$ as the equilibrium constant for the opening of the terminal pair without 


\section{- O- deoxyguanosine terminal G}

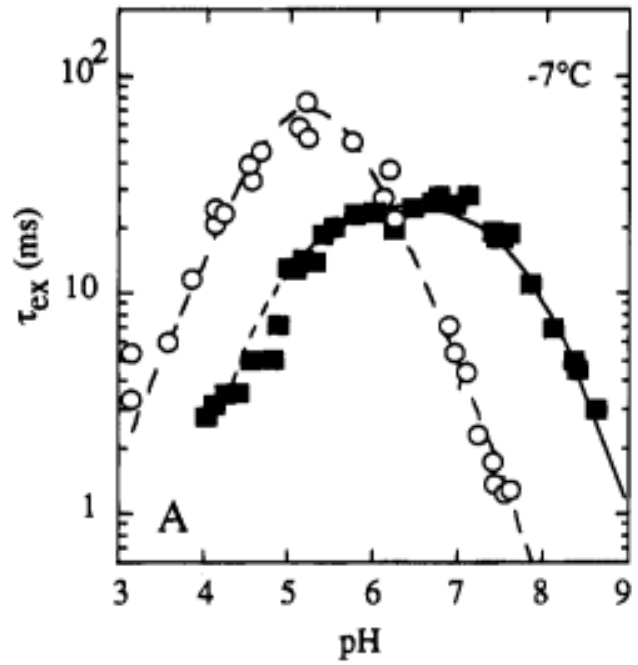

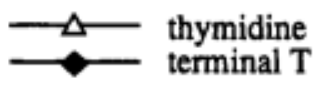

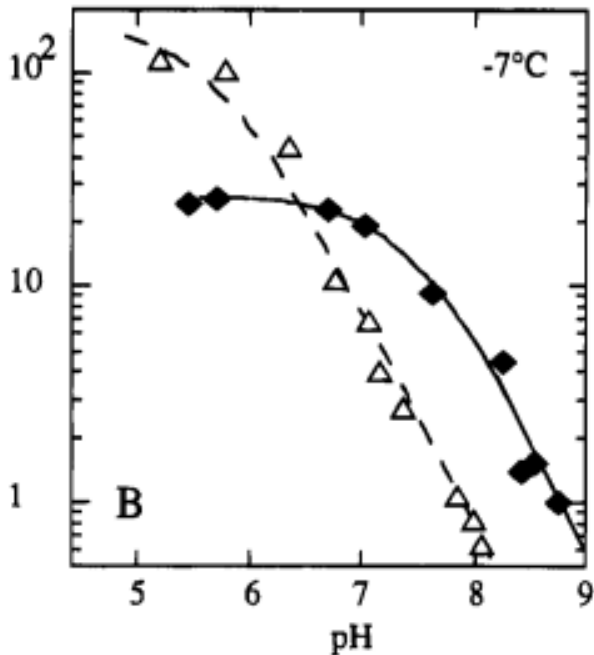

FIGURE 1: (A) Exchange time of the imino proton of the terminal G (G1, opposite C1) of the d(CGCGATCGCG) duplex and of deoxyguanosine $v s \mathrm{pH}$. At high $\mathrm{pH}$, when catalysis by $\mathrm{OH}^{-}$dominates, the exchange time of the terminal pair is larger than that of deoxyguanosine. At lower $\mathrm{pH}$, exchange is catalyzed by the intrinsic catalyst in the case of the terminal pair and by water in the case of deoxyguanosine. Conditions: $\mathrm{NaCl}, 200 \mathrm{mM}$; EDTA, $1 \mathrm{mM} ;-7^{\circ} \mathrm{C}$. The $\mathrm{pH}$ scale is inaccurate. (B) Exchange time of the imino proton of the terminal $\mathrm{T}$ of the d(TAGCGCTA) duplex and of thymidine $v s \mathrm{pH}$. Same conditions as in (A) except $\mathrm{NaCl}, 100 \mathrm{mM}$.

deprotonation when the second one is closed (the equilibrium c1c2 $\leftrightarrow$ olc2). The dissociation constant of the terminal pair, $K^{\prime}$, still conditional on the second pair being closed but including the two open states olc2 and $\mathrm{ol}^{-} \mathrm{c} 2$, is given by a relation analogous to (9):

$$
K^{\prime} \equiv\left(\mathrm{olc} 2+\mathrm{ol}^{-} \mathrm{c} 2\right) / \mathrm{clc} 2=K_{\mathrm{d}}(1 ; \mathrm{c} 2)\left[1+10^{\mathrm{pH}-\mathrm{p} K_{\mathrm{i}}}\right]
$$

The dissociation constant of the first pair is thus

$$
\begin{aligned}
& K_{\text {diss }}(1) \equiv \\
& \left(\mathrm{o} 1 \mathrm{c} 2+\mathrm{o} 1^{-} \mathrm{c} 2+\mathrm{o} 1 \mathrm{o} 2+\mathrm{o} 1^{-} \mathrm{o} 2+\mathrm{o} 1 \mathrm{o} 2^{-}+\mathrm{o} 1^{-} \mathrm{o} 2^{-}\right) / \\
& \mathrm{c} 1 \mathrm{c} 2=K^{\prime}\left(1+K^{\prime}\right)(11)
\end{aligned}
$$

The dissociation constant of the second pair is

$$
\begin{aligned}
K_{\text {diss }}(2) \equiv( & \left(0102+o 1^{-} \mathrm{o} 2+\mathrm{o} 102^{-}+\mathrm{ol}^{-} \mathrm{o} 2^{-}\right) / \\
& \left(\mathrm{o} 1 \mathrm{c} 2+\mathrm{o} 1^{-} \mathrm{c} 2+\mathrm{c} 1 \mathrm{c} 2\right)=K^{\prime 2} /\left(1+K^{\prime}\right)
\end{aligned}
$$

The values of $K_{\text {diss }}(1)$ and $K_{\text {diss }}(2)$ are close to $K^{\prime}$ and $K^{\prime 2}$, respectively, when $K^{\prime}$ is small.

\section{RESULTS}

\section{d(CGCGATCGCG)}

Imino Proton Exchange. The crucial observation is that exchange catalysis of the imino proton by $\mathrm{OH}^{-}$is very much less efficient for the terminal $\mathrm{G}(\mathrm{G} 1$, opposite $\mathrm{C} 1$ ) than for isolated deoxyguanosine in the same conditions. This is shown in Figure 1A which displays the exchange times of the imino proton in $\mathrm{G} \underline{1}$ and in deoxyguanosine. At high $\mathrm{pH}$, the dominant catalyst is $\mathrm{OH}^{-}$, as indicated by the -1 slope of the log plot. [Exchange catalysis by the deprotonated nucleoside (Guéron et al., 1987) would give the same slope, but this effect is ruled out at the concentrations used here]. Exchange catalysis by $\mathrm{OH}^{-}$is approximately 50 times slower for $\mathrm{G} 1$ than for deoxyguanosine. In the absence of further information, we cannot decide on the contribution of the closed state to exchange. At most, the efficiency of

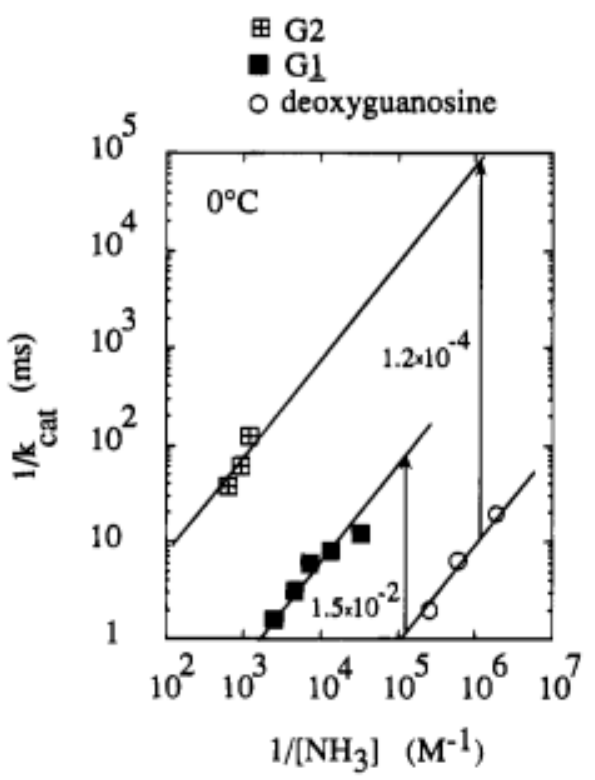

FIGURE 2: The contribution of ammonia to the exchange rate of the imino proton of $\mathrm{G} 1$ and $\mathrm{G} 2$ of the d(CGCGATCGCG) duplex and of deoxyguanosine. Conditions: $\mathrm{NaCl}, 200 \mathrm{mM}$; EDTA, $1 \mathrm{mM}$; $0{ }^{\circ} \mathrm{C}$.

exchange from the closed state, as compared to deoxyguanosine, is $1 / 50$. It could be much less if, as we expect, most of the exchange occurs from the open state.

In contrast to the high-pH situation, exchange at $\mathrm{pH} 6$ and below is faster from the terminal $\mathrm{G}$ than from deoxyguanosine. The explanation is simple: at the reduced $\mathrm{OH}^{-}$ concentration, other exchange processes take over. For deoxyguanosine, the dominant proton acceptor is now $\mathrm{H}_{2} \mathrm{O}$. For the terminal $\mathrm{G}$, exchange from the open state is catalyzed by an intrinsic catalyst, the imino nitrogen of the opposite cytidine, which is more efficient than $\mathrm{H}_{2} \mathrm{O}$ (Guéron et al., 1987; Guéron \& Leroy, 1992).

Figure 2 compares catalysis by ammonia of proton exchange in $\mathrm{G} 1, \mathrm{G} 2$, and deoxyguanosine at $0{ }^{\circ} \mathrm{C}$. The relative efficiency of the catalyst is indicated. These numbers 


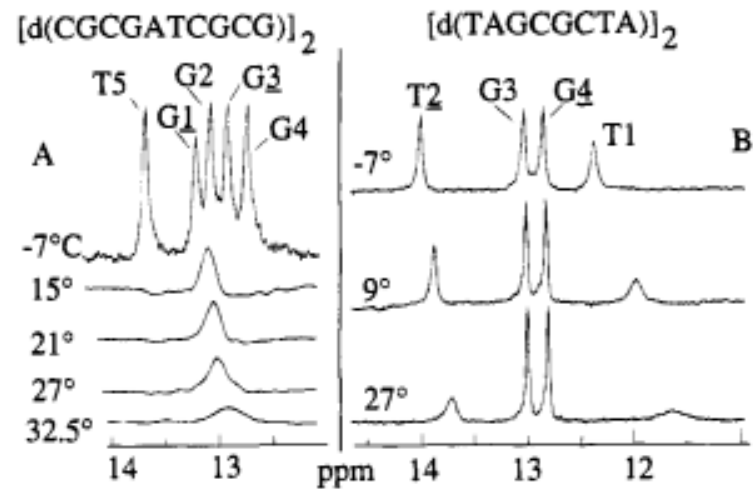

Figure 3: (A) Shift of the imino proton resonance of $\mathrm{Gl}$ of the $\mathrm{d}($ CGCGATCGCG) duplex $v s$ temperature. The resonance is in a fast-exchange situation between the positions corresponding to closed and open pairs. Exchange with water produces broadening but no shift. In all spectra except for the $-7{ }^{\circ} \mathrm{C}$ spectrum, all imino protons are saturated, except for the terminal one, which is therefore observable without interference, as described in Materials and Methods. The short acquisition time and Hamming filtering produce an instrumental broadening which is most simply determined by the line width of the methyl proton (not shown). The broadening assigned to exchange with water is $33,40,47$, and $97 \mathrm{~Hz}$ at 15 , 21,27 , and $32.5^{\circ} \mathrm{C}$, respectively. Conditions: $\mathrm{NaCl}, 200 \mathrm{mM}$; EDTA, $1 \mathrm{mM}$; chemical shift reference, $\mathrm{NMe}_{4} \mathrm{Cl}, 0.2 \mathrm{mM}$; $\mathrm{pH} 6.86$ (measured at $20^{\circ} \mathrm{C}$ ); $0^{\circ} \mathrm{C}$. (B) The imino proton spectrum of the d(TAGCGCTA) duplex $v s$ temperature, displaying the shift and broadening of the first and second thymidines. Conditions: $\mathrm{NaCl}$, $100 \mathrm{mM}$; EDTA, $1 \mathrm{mM}$; chemical shift reference, $\mathrm{NMe}_{4} \mathrm{Cl}, 0.2$ $\mathrm{mM} ; \mathrm{pH} 5.6$ (measured at $20^{\circ} \mathrm{C}$ ); $0{ }^{\circ} \mathrm{C}$.

are more reliable than those stated above for $\mathrm{OH}^{-}$catalysis, because the $\mathrm{pH}$ values at $-7{ }^{\circ} \mathrm{C}$ were not precisely known (see Theory).

The Dissociation Constant of the Terminal Pair. Two different measurements provide estimates of the dissociation constant, independent of each other and of exchange rate measurements.

(a) Derivation from the Chemical Shift of the Imino Proton vs Temperature. In Figure 3A, we present imino proton spectra for different temperatures. In order to measure the chemical shift of the imino proton of G1, we took advantage of the different relaxation times of the terminal imino proton and of the others, as described in Materials and Methods. The change in chemical shift with temperature is ascribed to the variation of the dissociation constant, in a fast-exchange situation between the closed and open states of the pair (see Theory). We have approximate values for the chemical shift in the open and closed pairs: for the open pair, the chemical shift should be close to that of deoxyguanosine, ca. $11.07 \mathrm{ppm}$ (not shown). For the closed pair, we use a trial value of the chemical shift (for instance, 13.25 ppm) which is close to that (13.2 ppm) observed at $-9{ }^{\circ} \mathrm{C}$ (Figure 4A), a temperature where the dissociation constant is small. Using eqs 5 and $10-12$ and the relation between $K_{\mathrm{d}}(1 ; \mathrm{c} 2)$ and the the thermodynamic parameters of base pair opening (the equilibrium $\mathrm{clc} 2 \leftrightarrow$ olc2),

$$
K_{\mathrm{d}}(1 ; \mathrm{c} 2)=\exp -((\Delta H-T \Delta S) / k T)
$$

we fit the data to values of $\Delta H$ and $\Delta S$.

Table 1 shows the results for three choices of the closedpair chemical shift. On the basis of the quality of the fit, judged both by eye and by the $\chi^{2}$ value, the first and third fits are unacceptable, while the second one (underlined in Table 1) is acceptable.

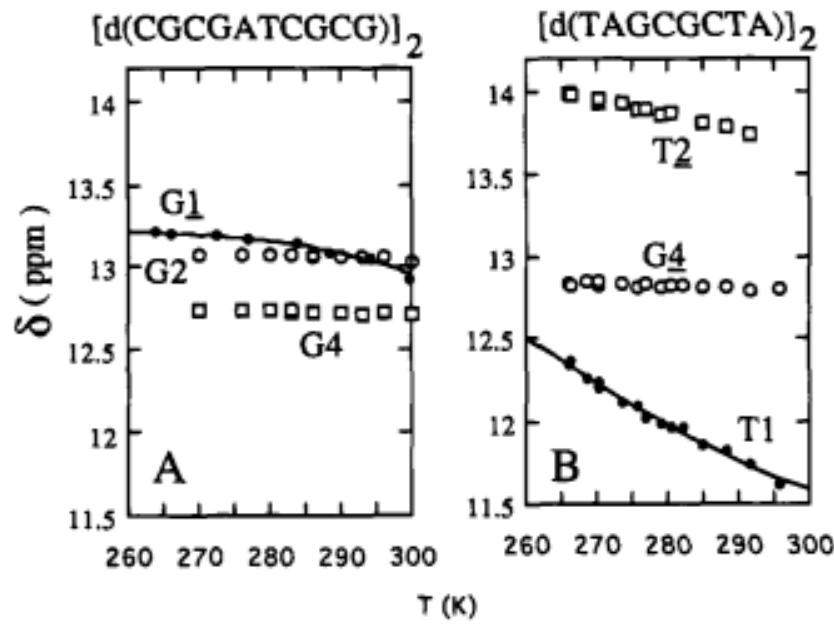

FIGURE 4: (A) Shift of the G1 imino proton line with temperature. The fitting procedure is described in the text. G2 and G4 are shown for comparison. A large shift of T5 (Leroy et al., 1988; not shown) is not due to base pair opening since there is no corresponding line broadening. Same conditions as in Figure 3A. (B) Shift of the T1 imino proton with temperature, fitted as described in the text, with $\mathrm{T} \underline{2}$ and $\mathrm{G} \underline{4}$ for comparison. Same conditions as in Figure 3B.

We get good fits of the chemical shift vs temperature for closed-pair shifts between 13.3 and $13.21 \mathrm{ppm}$ (Figure 4A). The corresponding values of $\Delta H$ are $28.8-49.2 \mathrm{~kJ} / \mathrm{mol}$, with midtransition temperatures of $360-337 \mathrm{~K}$.

By eq 12, the dissociation constant of the second pair should be no more than 0.025 across the experimental temperature range, and this justifies the use of a single chemical shift for the open pair, independently of temperature.

(b) Derivation from the Chemical Shift of the Aromatic Proton vs $\mathrm{pH}$. Figure 5A shows the aromatic region as a function of $\mathrm{pH}$ at $0{ }^{\circ} \mathrm{C}$. With increasing $\mathrm{pH}$, and up to $\mathrm{pH}$ 12.19 , the $\mathrm{G} 1 \mathrm{H} 8$ proton moves downfield, in a fastexchange situation. At higher $\mathrm{pH}$, the peak disappears due to the melting of duplex. The shift is plotted $v s \mathrm{pH}$ in Figure 6A (filled circles). It has two contributions: one from deprotonation which gives a $0.15 \mathrm{ppm}$ upfield shift, as shown by the monomer (Figure 6A, open circles), and one from pair opening whose sign and value depend on the location of the base when the pair is open.

We analyze the $\mathrm{pH}$ dependence of the shift as explained in Theory. The fit in Figure 6A is obtained with a dissociation constant of 0.008 , corresponding to an apparent $\mathrm{p} K$ shift of 2.1 units. The uncertainty is about $\pm 30 \%$, as judged by the goodness of fit. But there is further uncertainty, due to the crudeness of the model. For instance, at $\mathrm{pH} 12.19$, the computed open fraction is 0.76 for the terminal pair and 0.43 for the second one. Therefore, the approximation of a single value of the chemical shift of the open terminal pair across the experimental $\mathrm{pH}$ range is valid only if the chemical shift takes similar values when the second pair is open and when it is closed.

The $\mathrm{C} 1 \mathrm{H} 6$ peak behaves in similar fashion.

Dissociation Constant of the Second Pair. At $0{ }^{\circ} \mathrm{C}$ and ca. $\mathrm{pH} 6.8$, the dissociation constant of the terminal pair is small. Since that of the second pair should be smaller still, one trusts that exchange of the imino proton of the second pair takes place from the open state. The dissociation constant obtained from the rate of ammonia-catalyzed exchange (Figure 2) is $1.2 \times 10^{-4} / \alpha$. It is much larger than that of a typical internal G.C pair $\left(\approx 10^{-6}\right)$, showing that the dominant opening mode is indeed a fraying mode, which 
Table 1: Fitting the Chemical Shift of the Imino Proton to Opening of the Terminal Base Pair and Comparison with Calorimetry

\begin{tabular}{|c|c|c|c|c|c|c|}
\hline \multirow[b]{2}{*}{$\delta_{\text {closed }}(\mathrm{ppm})$} & \multirow[b]{2}{*}{ enthalpy $(\mathrm{kJ} / \mathrm{mol})$} & \multirow[b]{2}{*}{ entropy $(\mathrm{kJ} /(\mathrm{mol} \mathrm{K}))$} & \multirow[b]{2}{*}{$T_{n^{b}}^{b}(\mathrm{~K})$} & \multicolumn{2}{|c|}{$K_{\text {diss }}{ }^{e}$} & \multirow[b]{2}{*}{$\chi^{2}$} \\
\hline & & & & $0^{\circ} \mathrm{C}$ & $25^{\circ} \mathrm{C}$ & \\
\hline \multicolumn{7}{|c|}{$\left[5^{\prime}-\mathrm{d}(\text { CGCGATCGCG) }]_{2}\right.$} \\
\hline $13.21^{d}$ & 49.2 & 0.146 & 337 & 0.017 & 0.11 & 0.0046 \\
\hline $13.25^{e}$ & 38.0 & 0.110 & 345 & 0.031 & 0.14 & 0.0032 \\
\hline $13.30^{8}$ & $\overline{28.8}$ & 0.080 & 360 & 0.049 & 0.15 & 0.0038 \\
\hline calorimetry & $49.7^{\prime}$ & $0.116^{\prime}$ & 428 & $0.36 \times 10^{-3}$ & $2.2 \times 10^{-3}$ & \\
\hline \multicolumn{7}{|c|}{$\left[5^{\prime}-\mathrm{d}(\mathrm{TAGCGCTA})\right]_{2}$} \\
\hline$\geq 13.00$ & $\leq 22.8$ & $\leq 0.077$ & $\geq 296$ & $\geq 0.67$ & $\geq 2.2$ & $\approx 0.01$ \\
\hline calorimetry & $25.1^{f}$ & $0.071^{f}$ & 354 & 0.087 & 0.24 & \\
\hline
\end{tabular}

${ }^{a}$ Fit of enthalpy and entropy according to eqs 5 and $10-13$. The chemical shift of the imino proton in an open pair is set to $11.07 \mathrm{ppm}$ for $\mathrm{G}$ and $11.00 \mathrm{ppm}$ for $\mathrm{T}$. The shift assumed for the closed pair is indicated in the first column. ${ }^{b}$ Midtransition temperature $\left(T_{\mathrm{m}}=\Delta H / \Delta S\right)$. ${ }^{c} K_{\text {diss }}$ is computed by using eqs 10 and 11 from $K_{\mathrm{d}}(1 ; \mathrm{c} 2)$, which is obtained from the enthalpy and entropy by using eq $13 .{ }^{d}$ Visually poor fit. ${ }^{e}$ Visually good fit. ${ }^{\prime}$ Conditions: $1 \mathrm{M} \mathrm{NaCl}$, pH 7; from Breslauer et al. (1986).

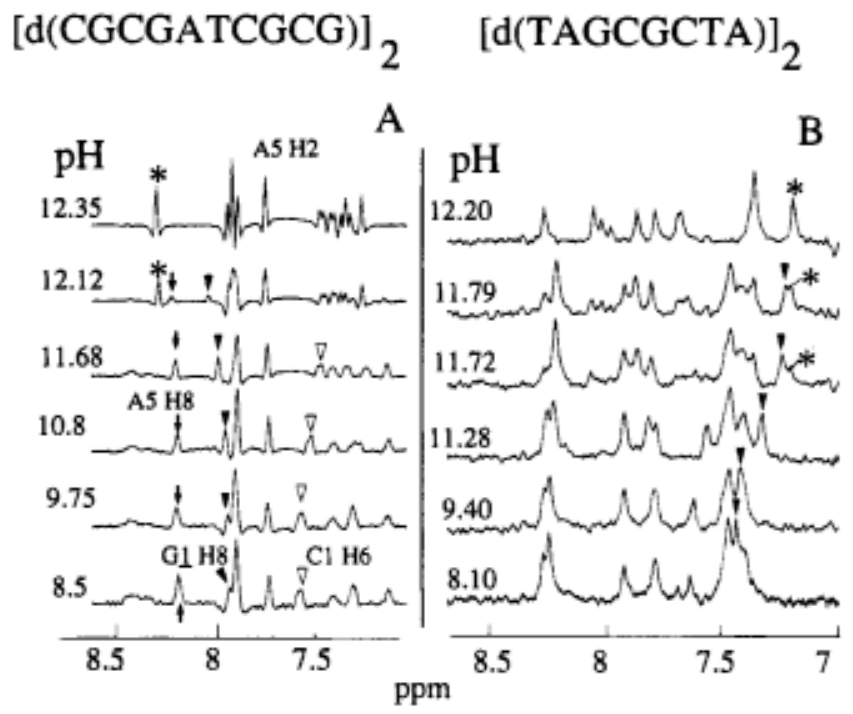

FIGURE 5: (A) Aromatic proton spectrum of the d(CGCGATCGCG) sequence $v s \mathrm{pH}$ (measured at $0^{\circ} \mathrm{C}$ ). The shift of the protons of the first pair corresponds to titration of base pair opening, in fast exchange. In contrast, melting of the duplex occurs in slowexchange conditions for all resonances. This is clear in the intensity changes of the $\mathrm{A} 5 \mathrm{H} 8$ lines $v s \mathrm{pH}$. These lines appear at 8.2 and $8.3 \mathrm{ppm}$ (asterisks), respectively, in the native ( $\mathrm{pH} 8.5$ ) and melted (pH 12.35) forms. Conditions: $\mathrm{NaCl}, 200 \mathrm{mM}$; EDTA, $1 \mathrm{mM} ; \mathrm{H}_{2} \mathrm{O}$, $90 \% ; \mathrm{NMe}_{4} \mathrm{Cl}, 0.2 \mathrm{mM}, 0^{\circ} \mathrm{C}$. (B) Aromatic proton spectrum of the d(TAGCGCTA) sequence vs pD. As in (A), the spectral situation is of fast exchange for base pair opening and slow exchange for duplex melting. This is apparent for the T1H6 proton. labeled with a solid arrowhead (native form) and an asterisk (melted form). Conditions: $\mathrm{NaCl}, 100 \mathrm{mM}$; EDTA, $1 \mathrm{mM} ; \mathrm{D}_{2} \mathrm{O}$ solution; $\mathrm{Me}_{4} \mathrm{Cl}, 0.2 \mathrm{mM}, 0{ }^{\circ} \mathrm{C}$.

can be analyzed as in the Fraying subsection of Theory. According to eq 12, the dissociation constant of the second pair should be close to the square of the dissociation constant of the terminal pair, for which we have two estimations, 0.019 and 0.008 . As discussed, $\alpha$ should not be far from 1 , and $1.2 \times 10^{-4}$ indeed falls between the squares of the two estimations.

Strand Separation at High $\mathrm{pH}$. At high $\mathrm{pH}$, a new spectrum appears, that of the single strand (Figure 5A). The switch from the low-pH to the high-pH spectrum takes place in a slow-exchange regime (Figure $5 \mathrm{~A}$ ), over one-half $\mathrm{pH}$ unit. This is clear in the case of the well-resolved A5H8 peak. With increasing $\mathrm{pH}$, there is a very slight shift of the native peak at $8.2 \mathrm{ppm}$. Starting from $\mathrm{pH} 11.73$, the intensity decreases and the peak of the melted species appears at 8.3 ppm. At $\mathrm{pH} 12.24$, the low-pH peak has disappeared.

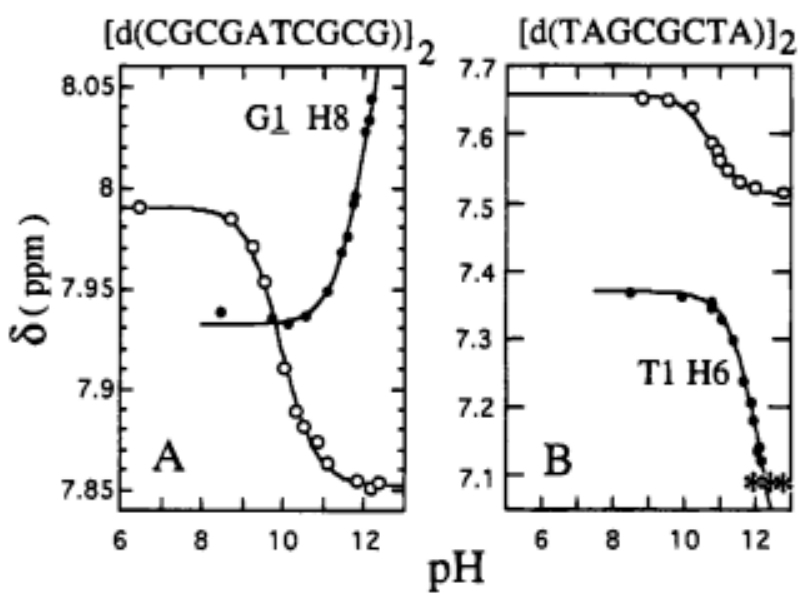

Figure 6: (A) Titration of the chemical shifts of G1H8 of the $\mathrm{d}(\mathrm{CGCGATCGCG)}$ sequence $(\mathbf{)})$ and of deoxyguanosine $(O)$. The fitting procedure is described in the text. The fit corresponds to a dissociation constant of 0.008 for the terminal pair at neutral $\mathrm{pH}$, which corresponds to an apparent $\mathrm{p} K$ shift of 2.1 units. The dissociation constant is 1.36 at $\mathrm{pH} \mathrm{12.19}$. Same conditions as in Figure 5A. (B) Titration of the chemical shifts of T1H6 of the d(TAGCGCTA) sequence ( $)$ and of thymidine $(O)$. Asterisks correspond to the chemical shift in the melted form. The fit corresponds to a dissociation constant of 0.1 for the terminal pair at neutral pH, and of 1 at $\mathrm{pH} 11.7$. Same conditions as in Figure $5 \mathrm{~B}$.

Except for poor resolution, the other resonances display the same behavior.

Bonds on the Rate of Exchange from the Closed State of the Terminal Pair. Even if exchange occurred exclusively from the closed state of the terminal pair, its rate would only be 0.015 times that for isolated deoxyguanosine in the conditions of Figure $2\left(0^{\circ} \mathrm{C}, \mathrm{pH} 7\right)$. Taking into acount the contribution of exchange from the open state, it could be much slower still. Indeed, the exchange contribution from ammonia catalysis in the open state can be estimated by eq 3. The data in Figure 2 show that exchange is not openinglimited; hence the rate of ammonia-catalyzed exchange is $\left(1+1 / K_{\text {diss }}\right) / \alpha k_{\mathrm{i}}$. Assuming $\alpha=1$, and with $K_{\text {diss }} \ll 1$, the predicted efficiency ratio with respect to isolated deoxyguanosine is simply $K_{\text {diss. }}$. Comparison of the two evaluations of the dissociation constant of the terminal pair, 0.019 and 0.008 , with the observed ratio, 0.015 , shows that exchange from the open state could account entirely for the observations. There is thus no evidence for any exchange from the closed state. We conclude that ammonia-induced exchange from the closed state of the terminal pair is at least 67 times 
Table 2: Comparative Values of the Base Pair Dissociation Constant $K_{\text {diss }}$ of the Terminal Base Pairs at $0{ }^{\circ} \mathrm{C}$

\begin{tabular}{|c|c|c|c|c|}
\hline \multirow[b]{2}{*}{ base pair } & \multicolumn{4}{|c|}{ method } \\
\hline & $\mathrm{ex}^{a}$ & $\delta(T)^{b}$ & $\delta(\mathrm{pH})^{c}$ & calorimetry ${ }^{d}$ \\
\hline $\begin{array}{l}{\left[5^{\prime} \text {-d(CGCGATCGCG) }\right]_{2}{ }^{e}} \\
\text { terminal } \\
\text { second } \\
{\left[5^{\prime} \text {-d(TAGCGCTA } f^{f}\right.}\end{array}$ & $\begin{array}{l}15 \times 10^{-3} \\
0.12 \times 10^{-3}\end{array}$ & $31 \times 10^{-3}$ & $8 \times 10^{-3}$ & $0.35 \times 10^{-3}$ \\
\hline terminal & 0.6 & $\geq 0.67$ & $0.05-0.2$ & 0.087 \\
\hline
\end{tabular}

${ }^{a}$ From the imino proton exchange rate, assuming that there is no exchange from the closed pair. ${ }^{b}$ From the imino proton shift with temperature. Same values as in Table $1 .{ }^{c}$ From the apparent $\mathrm{pK}$ shift of the imino proton, derived from the chemical shift of aromatic protons $v s$ pH. ${ }^{d}$ As in Table 1. From Breslauer et al. (1986), ${ }^{e} \mathrm{pH} 6.86 .{ }^{f} \mathrm{pH} 5.6$.

slower than from deoxyguanosine, and it could be much slower still.

\section{d(TAGCGCTA)}

This duplex was studied like the d(CGCGATCGCG) duplex (panels $\mathrm{B}$ of Figures 1 and 3-6). The rate of exchange catalysis by ammonia for the terminal $\mathrm{T}$ is 0.37 times that for thymidine at $0^{\circ} \mathrm{C}$. This value is the upper limit on the efficiency of exchange from the closed pair. Alternatively, if it corresponds to exchange from the open state, the dissociation constant $K_{\text {diss }}$ is 0.59 (eq 1 ).

The temperature dependence of the chemical shift (Figure 3B) provides only a lower limit, 0.67 (Table 1), for the dissociation constant at $0^{\circ} \mathrm{C}$. The evaluation based on the $\mathrm{p} K$ shift at $0^{\circ} \mathrm{C}$ is much smaller, and therefore incompatible with the two values just given (Table 2).

\section{DISCUSSION}

\section{Exchange from Closed Pairs}

The present results indicate that imino proton exchange from the closed terminal base pair of a DNA duplex is too slow to be observed. The most stringent limit is obtained in the case of the terminal $\mathrm{C} \cdot \mathrm{G}$ pair of the CGCGATCGCG duplex, the one with the smallest dissociation constant. Exchange from the closed pair is at least 67 times slower than from isolated deoxyguanosine, and it could be much slower. The experimental constraint is weaker for the terminal T*A pair, but there is no reason to believe that exchange from the closed state is faster than from terminal $\mathrm{C} \cdot \mathrm{G}$ pairs. Thus, a base pair with one face completely exposed to the solution provides no easy pathway for exchange of the imino proton.

The protection from exchange provided by the complementary base may be due to the involvement of the proton in a hydrogen bond or to the steric obstruction of favorable directions of attack by exchange catalysts. It is probably not due to a $\mathrm{p} K$ shift, since there is protection from the $\mathrm{OH}^{-}$ catalyst (Figure 1) as well as from ammonia. No model of proton exchange from a closed pair has been proposed, and there is therefore no theoretical estimate of the protection factor. One expects that these conclusions apply also to base pairs of RNA.

Another case of an exposed base pair is provided by a "kink", the defect in a nucleic acid duplex at the point where the direction of the duplex axis changes suddenly, with unstacking but not opening of base pairs. Existence and prevalence of kinks are not known. Our results show that kinking would not enhance imino proton exchange directly, But it might do so indirectly, by increasing the dissociation constant of pairs at the kink position. Similar considerations apply to pairs next to an abasic site.
Exchange from the closed state should also be considered in wobble pairs such as G.T whose imino protons are sterically accessible sideways, from within the plane of the pair. Exchange from such pairs within a duplex is efficiently catalyzed by proton acceptors (Leroy et al., 1985; Moe \& Russu, 1992). Available measurements cannot distinguish between exchange from the closed state and exchange from the open state of a pair whose dissociation constant is large and whose lifetime is too short to be distinguished from zero. Since the imino protons of wobble pairs are accessible from the grooves, in directions within the base plane rather than perpendicular to it as for a terminal Watson-Crick pair, the present work provides no indication on the rate of exchange from the closed wobble pair. An indication is provided by comparison with the internal amino proton of a WatsonCrick pair or of a $\mathrm{C}^{-} \mathrm{C}^{+}$pair, since these are also accessible in plane, from the grooves. In the i-motif geometry, exchange of the internal amino protons of a $\mathrm{C}^{\cdot} \mathrm{C}^{+}$pair is hundreds of times slower than exchange of the external amino proton or of amino protons of the hemiprotonated nucleoside (Leroy et al., 1993), suggesting that exchange from the closed state is strongly inhibited.

\section{The Open State of Terminal Pairs.}

As discussed above, we expect that the upper limit on exchange from the closed state of the terminal C. $\mathrm{G}$ pair (1/ 67 times the rate for the isolated nucleoside) is generally valid. Exchange from the closed state can therefore be ignored if the observed exchange rate is much larger than this upper limit. Given the lack of evidence for any exchange process from the closed state, we now assume that such a process is negligible in all cases.

Efficiency of Exchange from the Open State. The comparison of the exchange rate (relative to that of the isolated nucleoside) and of the independently measured dissociation constant provides a direct evaluation of $\alpha$, the coefficient which reflects the efficiency of the catalyst for exchange from the open state (eq 2).

In the terminal pair of the d(TAGCGCTA) duplex, the efficiency of exchange catalysis by ammonia relative to the nucleoside is 0.37 . By eqs $1-3$, this ratio is equal to $\alpha /$ ( 1 $+1 / K_{\text {diss }}$ ). From the imino proton chemical shift study, we estimate that the dissociation constant is larger than 0.67 (Table 1). Hence

$$
0.37<\alpha<0.37(1+1 / 0.67)
$$

This puts $\alpha$ between 0.3 and 0.92 , not far from unity, considering that, a priori, any value between $10^{-2}$ and 10 would not have been astonishing.

In the case of the terminal $\mathrm{C} \cdot \mathrm{G}$ pair, the fair agreement of the three values of the dissociation constant was used to 
provide a limit on exchange from the closed state, assuming that $\alpha$ is close to 1 . Therefore, this measurement cannot provide a value of $\alpha$. Still, we note that if we assume that there is no exchange from the closed state, the approximate agreement between the three values of $K_{\text {diss }}$ implies that $\alpha$ is close to 1 .

These are the first direct evaluations of $\alpha$, based on independent estimates of the exchange rate and the dissociation constant. They should of course be interpreted with caution, considering in particular the discrepancies between the different evaluations of the dissociation constant (Table 2). Nevertheless, they suggest that external catalysts interact efficiently with the imino proton in the open state of terminal pairs. A similar result was obtained earlier for internal base pairs, but the argument was indirect, lacking an exchangeindependent measurement of the dissociation constant (Gueron et al., 1990; Guéron \& Leroy, 1992).

Intrinsic Catalysis. Intrinsic catalysis of exchange is due to concerted proton transfer across a water bridge to the imino nitrogen of the opposite base in the open state. For the terminal pair of the duplexes studied here, measurements of exchange in the absence of added catalyst (the plateau in Figure 1) indicate that the efficiency of intrinsic catalysis is equivalent to $0.02 \mathrm{mM}$ ammonia for the terminal $\mathrm{C} \cdot \mathrm{G}$ pair and $0.004 \mathrm{mM}$ for the terminal $\mathrm{T} \cdot \mathrm{A}$ pair at $0{ }^{\circ} \mathrm{C}$. This is much less than in the case of internal pairs, for which the efficiency is $\approx 0.75-3 \mathrm{mM}$ ammonia for G.C pairs and $\approx 0.14-1.1 \mathrm{mM}$ for $\mathrm{A} \cdot \mathrm{T}$ pairs, with little effect of temperature [Figure 3 in Gueron et al. (1987)]. The efficiency is also low for the second C.G pair, as can be seen from an earlier $\mathrm{pH}$ titration at $25^{\circ} \mathrm{C}$ [Figure 5 in Guéron and Leroy (1992)].

This suggests that terminal base pairs open up more widely than internal pairs: two or more water molecules would be required to span the $\mathrm{N}-\mathrm{H} / \mathrm{N}$ distance, rather than presumably one in internal pairs. This would slow down proton transfer across the water bridge. If this interpretation is confirmed, the effectiveness of intrinsic catalysis will provide a new method for the study of fraying.

Thermodynamics of Base Pair Opening. Table 2 presents values of the dissociation constants based on exchange, on the temperature dependance of the imino proton chemical shift, and on the imino proton $\mathrm{p} K$ shift. We believe that the derivation of the dissociation constant from the exchange measurement is the most trustworthy. The derivation involves few relatively straigthforward assumptions: absence of exchange from the closed state and comparable effects of external catalysts on the open state and on the isolated nucleoside (i.e., $\alpha \approx 1$ ). Arguments for these assumptions have been given above.

The dissociation constants at $0{ }^{\circ} \mathrm{C}$, derived from exchange, are presented in Table 2, column "ex". One notes the larger dissociation constant of the terminal T•A pair. The dissociation constant of the second $\mathrm{G} \cdot \mathrm{C}$ pair is close to the square of that of the first pair, as expected from a simple model (eqs 10-12).

In the case of the terminal C.G pair the dissociation constant derived from the imino proton shift with temperature, column " $\delta(T)$ ", is within a factor of 2 of the exchange value, and this is also true for the value derived from the apparent $\mathrm{p} K$ shift of the imino proton, column " $\delta(\mathrm{pH})$ " (Table
2). We consider that the values from the three methods are in agreement.

With the thermodynamic data derived from the effect of temperature on the chemical shift, one expects a dissociation constant in the range of 0.14 for the terminal $C \cdot G$ pair at 25 ${ }^{\circ} \mathrm{C}$ (Table 1). This would imply a value of 0.02 for the second $\mathrm{G} \cdot \mathrm{C}$ pair, much larger than the values derived previously from catalyzed exchange: $(0.15-0.3) \times 10^{-3}$ (Guéron et al., 1990; Guéron \& Leroy, 1992).

For the terminal T•A pair, the fit of the temperature shift provides only a lower limit for the dissociation constant. This limited information is in near agreement with the exchange value. The value derived from the $\mathrm{p} K$ shift is much smaller. The proposed interpretation of the $\mathrm{p} K$ shift may well be oversimplified.

Lastly, we have listed in Tables 1 and 2 the thermodynamic data for base pair opening derived from the calorimetry of melting of oligodeoxynucleotide duplexes (Breslauer $e t$ al., 1986), although it is not obvious that they are directly applicable to fraying. The enthalpy and entropy values are roughly in the same range, and both sets of data show a greater stability of the terminal $5^{\prime}-\mathrm{d}(\mathrm{CG})$ sequence as compared to $5^{\prime}$-d(TA). But the dissociation constants derived from the calorimetry values are in general much smaller than those measured in the present study (Tables 1 and 2).

Beyond the valuable results discussed in the last section, the existence of discrepancies may serve as a reminder that much remains unknown in the thermodynamics of fraying, and that the major result of the present work is the inhibition of imino proton exchange from the closed state of terminal pairs.

\section{REFERENCES}

Breslauer, K. J., Frank, R., Blöcker, H., \& Marry, L. A. (1986) Proc. Natl. Acad. Sci. U.S.A. 83, 3746-3750.

Eigen, M. (1964) Angew. Chem., Int. Ed. Engl. 3, 1-19.

Guéron, M., \& Leroy, J. L. (1992) in Nucleic Acids and Molecular Biology, (Eckstein, F., \& Lilley, D. M. J., Eds.) Vol. 6, pp 1-22, Springer-Verlag, Berlin, Heidelberg.

Guéron, M., \& Leroy, J.-L. (1995) Methods Enzymol. (in press). Guéron, M., Kochoyan, M., \& Leroy, J. L. (1987) Nature 328, 8992.

Guéron, M., Charretier, E., Hagerhorst, J., Kochoyan, M., Leroy, J. L., \& Moraillon, A. (1990) in Structure \& Methods (Sarma, R. H., \& Sarma, M. H., Eds.) Vol. 3, pp 113-137, Adenine Press, Schenectady, NY.

Guéron, M., Plateau, P., \& Decorps, M. (1991) Prog. Nucl. Magn. Reson. Spectrosc. 23, 135-209.

Kochoyan, M., Leroy, J. L., \& Guéron, M. (1990) Biochemistry $29,4799-4805$.

Leroy, J. L., Bolo, N., Figueroa, N., Plateau, P., \& Guéron, M. (1985) in Biological Structure and Dynamics, Vol. 2, pp 915939, Adenine Press.

Leroy, J. L., Charretier, E., Kochoyan, M., \& Guéron, M. (1988a) Biochemistry 27, 8894-8898.

Leroy, J. L., Kochoyan, M., Huynh-Dinh, T., \& Guéron, M. (1988b) J. Mol. Biol. 200, 223-238.

Leroy, J. L., Gehring, K., Kettani, A., \& Guéron, M. (1993) Biochemistry 32, 6019-6031.

Moe, J. G., \& Russu, I. M., (1992) Biochemistry 31, 8421-8428.

Morris, G. A., \& Freeman, R. (1963) J. Magn. Reson. 29, 433462.

Patel, N., Bergman, J., \& Graslund, A. (1991) Eur. J. Biochem. 197, 597-604. 